\title{
UNA EXPERIENCIA CONTADA: LA ENSEÑANZA DEL PORTUGUÉS COMO LENGUA EXTRANJERA A COLOMBIANOS EN BOGOTÁ
}

\author{
Roanita Dalpiaz \\ Especialista en Desarrollo de Materiales Didácticos \\ Instituto de Cultura Brasil Colombia- IBRACO \\ ro_popa@hotmail.com \\ Luciana Stanzani \\ Candidata a Magíster en Educación con énfasis en Didáctica de Lenguas Extranjeras \\ Instituto de Cultura Brasil Colombia- IBRACO \\ stanzani.luciana@gmail.com
}

Resumen

Este artículo trata acerca del papel de un profesor extranjero en la enseñanza de un idioma extranjero a partir de la experiencia docente de dos brasileñas profesoras de portugués en un contexto de no inmersión en la ciudad de Bogotá. Constituye una conguración de los perles tanto del estudiante como del maestro inmerso en la enseñanza-aprendizaje del idioma portugués teniendo presente la relevancia sociocultural de la enseñanza de esa lengua, ya que ella hace parte de la construcción de la propia identidad de los brasileños como constituidores de una nación.

Palabras clave: portugués, lengua extranjera, enseñanza, profesor

\section{Summary}

This article is about the role of a foreign teacher in teaching Portuguese as a foreign language. It tells the experience of two Brazilian Portuguese teachers in the non - immersion context in the city of Bogota. It is a conguration of the proles of both the student and the teacher engaged in the teaching and learning processes of Portuguese, bearing in mind the cultural relevance of the language teaching, as it is part of the construction of identity of Brazilians as important part of their nation.

Key words: Portuguese, foreign language, teaching, tea-

\section{Introducción}

El objetivo de este trabajo es explorar un cuestionamiento acerca del papel del profesor de lengua extranjera, a partir de la experiencia como profesoras de portugués como Lengua Extranjera (PLE) a colombianos. Para cumplir con este objetivo, se desarrollará un perl de los estudiantes del Instituto de Cultura Brasil Colombia (IBRACO) y también se planteará una discusión, sobre la formación del profesor de PLE.

\section{El portugués para extranjeros: un breve panorama de su trayectoria}

Es importante decir que la enseñanza del idioma portugués como segunda lengua en

Ella tiene una larga historia de casi cinco siglos que se extiende desde los primeros esfuerzos de los religiosos jesuitas por la enseñanza del portugués a miembros de naciones indígenas (Almeida Filho, 1992). No obstante, no hay estudios e interpretaciones abundantes de esa tradición, porque no hay conciencia de su existencia, como tampoco de la importancia de estudios de cuestiones relacionadas con ellas. Desde este punto de vista es innegable, por lo tanto, la relevancia sociocultural de la enseñanza de

de la construcción de la propia identidad de los brasileños como constituidores de una nación. Así, estudiar y conocer esta tradición puede signicar conocer y comprender mejor la cultura y el carácter del pueblo brasileño.

Según apunta Gomes de Matos (1989), uno de los profesionales precursores en el trabajo de divulgación e institucionalización de esta 
área de enseñanza e investigación, el portugués como lengua extranjera empezó a desarrollarse a mediados de la década de los sesenta. De acuerdo con Almeida Filho (1992), a partir de la década de los sesenta y durante los años setenta se constató un mayor crecimiento de cursos de portugués y producción de materiales didácticos en instituciones en el exterior.

Ese aumento de interés en el idioma podría explicarse por algunas razones básicas. La primera de ellas se relaciona con el hecho que, con el desarrollo industrial de Brasil, se pasa a tener un mayor número de extranjeros que van a este país a trabajar, a través de empresas multinacionales interesadas en intercambiar, importar y exportar tecnología. Así, hubo un crecimiento continuo de la búsqueda de clases de portugués para extranjeros, principalmente en los grandes centros urbanos, se estimuló el interés de los profesores y también la proliferación de escuelas privadas que ofrecían cursos en esa especialidad.

La segunda razón se reere al aumento de los intercambios internacionales entre universidades brasileñas y del exterior, que aumentó también el número de personas interesadas en estudiar en el país, lo que llevó a las universidades nacionales a la creación de cursos regulares de PLE. Con ello creció, por lo tanto, la demanda de profesionales involucrados en el área de la enseñanza.

La tercera razón para el incremento del interés por el área de PLE sería la implantación del MERCOSUR por los gobiernos de Brasil, Argentina, Paraguay y Uruguay, que signica ampliación y mayor facilidad en las relaciones económicas, comerciales y de intercambio cultural con los países vecinos del sur de América Latina.

Se puede decir que la tradición de la enseñanza del portugués como lengua extranjera, aunque sea reciente, se aanzó desde de los años ochenta, al expandirse en áreas potencial- mente prósperas como la que se reere al mercado de trabajo y la del sector de la enseñanza.

\section{La enseñanza del portugués para extranjeros en la ciudad de Bogotá}

En primer lugar, hay que explicar el contexto de este tema de trabajo. Somos profesoras de portugués como lengua extranjera en el Instituto de Cultura Brasil Colombia (IBRACO) con sede en Bogotá. IBRACO es una entidad adscrita a la embajada de Brasil, que se dedica a la enseñanza del portugués desde 1996.

El IBRACO es el único organismo en Colombia autorizado por el Gobierno de Brasil para aplicar el examen de prociencia de la lengua portuguesa, Celpe-Bras ${ }^{1}$, desarrollado por el Ministerio de Educación de Brasil, aplicado allí y en otros países, como requisito para que el estudiante extranjero acceda a las becas que otorga anualmente el gobierno de ese país. Sin embargo, es oportuno mencionar también que en Medellín, EAFIT realiza dicha prueba.

En el Instituto trabajamos con un cuerpo docente básicamente conformado por profesores brasileños de diferentes áreas del conocimiento. El equipo está conformado por sociólogos, sioterapeutas, administradores, odontólogos, fonoaudiólogos y licenciados en lengua portuguesa, entre otros.

La población más signicativa del Instituto son estudiantes universitarios ya graduados que pretenden acceder a las becas otorgadas por el gobierno brasileño para las universidades de Brasil. Sin embargo, también hay estudiantes amantes de la cultura de Brasil, así como profesionales de distintas áreas en busca de mejores oportunidades laborales.

Teniendo en cuenta que el examen CelpeBras es de naturaleza comunicativa, es decir, evalúa la capacidad de uso de la lengua por medio de cuatro producciones escritas, en las

1 Para saber más sobre el examen, consulte http://celpebras.inep. gov.br/inscricao/ 
cuales el estudiante se enfrenta con diferentes géneros textuales (cartas, e-mail, folleto y artículos entre otros) e interlocutores, para vericar el dominio de la lengua en contextos formales e informales.

Para que el estudiante logre su objetivo de ser aprobado en el examen mencionado, IBRA$\mathrm{CO}$ ha trabajado con una metodología que se llama metodología híbrida (explicada más adelante), lo que se justica por lo novedosas que son todavía las teorías acerca de la enseñanza de portugués para extranjeros. Según José Carlos Almeida Filho (1997, p. 7), uno de los estudiosos que más se ha destacado en el área, "la enseñanza de portugués a hablantes de otras lenguas vive en una fase de consolidación institucional y de incremento de investigaciones en Brasil" (traducción nuestra). Aunque la lingüística aplicada a la enseñanza de portugués haya empezado en los sesenta (Almeida Filho, 1997), hoy se siente que se forman las bases para la enseñanza de tal idioma. Aparte de esto, el hecho de que estemos fuera del contexto de investigación de las universidades brasileras nos lleva a estudiar basadas en nuestra propia experiencia, o en la experiencia de otros académicos que trabajan allá.

De acuerdo a lo presentado arriba, el curso de portugués de IBRACO está destinado a jóvenes y adultos. Se compone de siete niveles, de 50 horas cada uno, y se ofrece como intensivo (entre semana) o los sábados. En el curso se trabaja con un libro didáctico, "Falar...Ler...Escrever... Portugues um curso para estrangeiros" (Sao Paulo, 2009) que integra el principal instrumento del método tradicional con la sistematización de las estructuras básicas de la lengua mediante la repetición de ejercicios escritos, básicamente, y con materiales de apoyo para cada nivel. Éstos fueron desarrollados por los propios docentes y están orientados al público especíco del Instituto, en una forma de trabajar con un abordaje comunicativo de la lengua por medio de actividades que incluyen lectura, video y música, así como producciones de textos orales y escritos.
Se entiende que, para la enseñanza de portugués a estudiantes que hablan español, no se puede aplicar una metodología únicamente comunicativa, porque eso podría hacer, a nuestro modo de ver, que el estudiante cayera en un estado de ínter lengua en el cual no alcanzara su total prociencia en la lengua portuguesa. De acuerdo con Almeida Filho (2001, p. 19),

"Hay ventajas indubitables en esa proximidad, especialmente si están combinadas con trazos afectivos como capacidad de riesgo, seguridad o también extroversión. Sin esos atributos, es de esperarse que ocurran las tentativas de obtención de uidez y de disponibilidad de vocabulario cuyo aparente (y posible) medio-éxito lleva a estancar la ínter lengua en niveles bajos de producción denominados popularmente de portuñol". (Traducción nuestra).

De acuerdo con esto, en la enseñanza de portugués a un hispano-hablante lo mejor es establecer las fronteras de las diferencias, para que el estudiante verdaderamente tome consciencia de que no se trata de la misma lengua. Sin duda, las semejanzas ayudan a que el proceso de aprendizaje sea más rápido, en comparación con estudiantes de otras lenguas.

\section{Resultados}

Dentro de ese universo aquí mencionado, podríamos cuestionarnos acerca del papel del profesor de PLE, especícamente en un contexto de no inmersión, y también sobre cuál sería su formación adecuada o esperada.

Los profesionales que llegan a IBRACO no son necesariamente de carreras relacionadas con las lenguas. Con este equipo interdisciplinario es viable pensar la lengua como un conjunto, en su contexto global, incluso en lo que se reere a la adquisición de vocabulario, sin limitar el aprendizaje a un grupo de reglas. Habida cuenta que los propios profesionales que estudiaron lenguas o lingüística no han tenido 
una preparación especíca para la enseñanza de su lengua materna a extranjeros, y, que este tema es muy reciente y todavía hay muchas universidades brasileñas que no lo han puesto en el currículo de sus cursos de lengua portuguesa, el Instituto elaboró un curso de formación de profesores, en el cual se habla de la metodología híbrida, y sobre algunos métodos que los profesores con más experiencia han aplicado con resultados satisfactorios.

Teniendo en cuenta al estudiante promedio del Instituto, esto es, aquel que habla español y por eso tendrá más facilidad en comunicarse, lo que como ya decimos puede caer en el riesgo de comunicarse con el "portuñol", pensamos que trabajar únicamente con un abordaje comunicativo no sería la mejor opción para él, porque no le daría la oportunidad de sistematizar las diferencias que hay entre el español y el portugués, que, en nuestra opinión, pueden ser resueltas con muchas propuestas que traen los libros más estructurales. También, tenemos que estar preparadas para este estudiante adulto, que estudia basado en la comprensión y que anhela saber el porqué deben actuar de una o otra manera, establecido en cada momento una relación con su lengua materna (Kunzendor , 1997).

Especícamente en la enseñanza de lenguas próximas, como es el caso del portugués y el español, creemos que trabajar con la gramática por la gramática puede ser una manera para que el estudiante, además de sistematizar las diferencias entre las dos lenguas, pueda hacer una reexión sobre su propia lengua.

Además concordamos con Lombello (1989, p. 45) en lo que se reere a la importancia del contenido gramatical en el aprendizaje de una lengua:

"Tanto por cuestiones de orden práctico como por la consideración de la arbitrariedad del signo lingüístico y de la necesidad del apoyo de sistematización al aprendiz, un programa general de enseñanza de una lengua extranjera para adultos debe ser orientado gramaticalmente, es decir, que debe tener una base gramatical organizada progresivamente en la cual se prevea una complementación incidental y variable a lo largo del desarrollo particular de cada programa". (Traducción nuestra).

El curso de formación de profesores también es el momento en el cual se reexiona sobre el papel del profesor de portugués como lengua extranjera a hispano hablantes. Es muy importante que el profesor conozca la lengua materna de sus estudiantes para poder entenderlos mejor en las dicultades presentadas. No podemos aceptar el posicionamiento de ciertas escuelas que deenden el principio de que, para enseñar portugués a extranjeros, basta ser un hablante nativo; hay que conocer también la cultura brasilera y actualizarse constantemente. En lo que se reere al uso de estructuras de la lengua hablada, un profesor que no esté en contacto constante con su país y con lo que se hace en la actualidad puede caer en el equívoco de enseñar expresiones coloquiales, por ejemplo, que ya no se usan o que son formas muy básicas, con las cuales el estudiante tendría dicultad de expresarse en el idioma en cuestión. Por eso, la formación pedagógica adecuada y las capacitaciones permanentes son fundamentales para la ecacia de cualquier curso.

Es evidente que no se postula aquí que el profesor deba ser del área de lenguas, pero es necesaria una preparación profesional, pues el éxito del aprendizaje de una lengua está relacionado sobretodo con la aplicación de nuevas teorías y con la capacidad de probar y de evaluar nuevos métodos.

\section{Conclusiones}

Por lo antes enunciado surge la necesidad de un curso de formación de profesores, para que los diferentes profesionales puedan actuar en el campo de la educación del PLE. El éxito 
del trabajo está en la aplicación adecuada del método y también en la constante actualización profesional, que posibilite al profesor un perfecto desempeño de sus funciones.

El profesor tiene un papel fundamental en la formación de un estudiante de lenguas extranjeras. Con tantos recursos tecnológicos con los cuales éste puede hoy en día aprender una lengua, sentimos que somos sus orientadores, pero sin contar con un contexto de inmersión, creemos en un profesor orientador

pues es con él que el extranjero mantiene su primer contacto y, consecuentemente, buscará orientaciones o informaciones sobre cómo, dónde y cuándo, es decir, se va a enterar, informar, conando, sobretodo, en sus recomendaciones [...] puesto que es él quien hará el puente entre el individuo extranjero y el nuevo mundo." (Souza, 2010, p. 105 - Traducción nuestra).

Porque al aportarles un poco de nuestra experiencia creemos que cuando se menciona el habla también se menciona la cultura. Dentro de ese contexto, el de un instituto cultural en el extranjero, una de las más ecaces cercanías a la cultura de Brasil es por medio de los profesores y de los programas, por ejemplo, que en el Instituto en cuestión se promueve para divulgar la cultura brasileña. En IBRACO se programa los "martes culturales", en los que se realizan presentaciones musicales, exposiciones, charlas con especialistas de diversas áreas como literatura, música y política.

En cada nivel, el estudiante puede aprender de la gramática básica a la avanzada, pero también desde la culinaria hasta la literatura de Brasil. Hay una programación de contenidos relacionados con la geografía y con la cultura de cada región de Brasil y el aporte del profesor es fundamental. IBRACO cuenta con profesores de muchos lugares de Brasil, lo que también favorece el contacto con la diversidad cultural existente en dicho país, que se sale de lo conocido internacionalmente a través de los medios o del turismo, por ejemplo. Aun con el hecho de que estemos en un contexto de no inmersión, es posible decir que los estudiantes del Instituto salen de sus cursos con un bagaje teórico con respecto a Brasil, su cultura y su historia.

Muchas veces por las películas, por la música y por todo este aparato que involucra la cultura y sus viajes a Brasil, el estudiante espera del profesor un lenguaje parecido al coloquial. Lo que se da en el Instituto, sin embargo, es un lenguaje más cercano a la lengua culta, ya que creemos que el tránsito de la lengua culta a la coloquial es mucho más fácil que de la coloquial a la lengua culta.

Además de orientar al estudiante en el aprendizaje de la lengua (entendemos que la cultura también hace parte de eso), el profesor es quien condiciona y motiva a sus estudiantes, ayudándolos a superar sus momentos difíciles.

Con respecto a ese contexto extranjero en el cual la lengua de los estudiantes es una lengua extranjera para los profesores, es posible decir que estar fuera del lugar de origen nos estimula a la reexión. Se analiza mucho más lo que pasa con el idioma y sobretodo con la cultura desde un contexto exterior. Esa abstracción nos hace trabajar todo el tiempo con el análisis de la comparación y nos estimula mucho a pensar en el otro y en la diferencia con el otro: la otra cultura, el otro idioma, el otro folclor y la otra música.

Finalmente, se enfatiza que nuestro objetivo, como profesoras del idioma portugués en Colombia, es hacer que el estudiante se acerque, a pesar de que no esté en un contexto de inmersión, a la cultura brasileña. Sea cual sea el método elegido para dictar un curso de portugués, es importante resaltar en que se aprende una lengua a través de la práctica constante de la misma. No existen fórmulas 
perfectas y absolutas, pues la lengua se caracteriza por su dinamismo. En este sentido, el mejor camino al aprendizaje es el de presentar propuestas innovadoras para la enseñanza en el momento actual, dejando abierta la posibilidad para nuevas modicaciones en futuro.

\section{REFERENCIAS}

Almeida Filho, José Carlos P. de; Lombello, Leonor C. (orgs.). (1997)O ensino de português para estrangeiros: pressupostos para o planejamento de cursos e elaboração de materiais. Campinas: Pontes.

Almeida Filho, José Carlos P. de. (1992). Relatório de Pesquisa: projeto interação e relevância no ensino de línguas Campinas: Unicamp.

Almeida Filho, José Carlos P. de. (2001). Português para estrangeiros interface com o espanhol. En: Almeida Filho, José Carlos P. de. (orgs.). Uma metodologia especíca para o ensino de línguas próximas? Campinas: Pontes.
Gomes de Matos, F. (1989). "Quando a prática precede a teoria”. En: Almeida Filho, José Carlos P. de.; Lombello, Leonor C. (orgs.). O ensino de português para estrangeiros: pressupostos para o planejamento de cursos e elaboração de materiais. Campinas: Pontes. Pp 11-17.

Lombello, Leonor C. (1989). "Quando a prática precede a teoria”. En: Almeida Filho, José Carlos P. de; Lombello, Leonor C. (orgs.). A anterioridade do lingüístico no planejamento de programas de ensino de segunda lengua. Campinas: Pontes. P 45.

Kunzendor , Júlia Cristina. Considerações quando ao ensino de português para estrangeiros adultos. En: Almeida Filho, José Carlos P. de.; Lombello, Leonor C. (orgs.). O ensino de português para estrangeiros: pressupostos para o planejamento de cursos e elaboração de materiais. Campinas: Pontes, 1997.

Souza, Ana Paula Kurpan de. O ensino de língua Portuguesa já foi além do limite: uma discussão teórica e metodológica sobre o ensino de PLE. Disponible en: http:// www.letras.puc-rio.br/publicacoes/ccci/Textos $\% 20$ revisados/0\%20Ensino\% 20de\%20L\%C3\%ADngua\%20 Portuguesa\%20foi\%20al\%C3\%A9m\%20do\%20limite. pdf. Acceso: 4 mayo 2010. 


\title{
UNA EXPERIENCIA CONTADA: LA ENSEÑANZA DEL PORTUGUÉS COMO LENGUA EXTRANJERA A COLOMBIANOS EN BOGOTÁ
}

\author{
Roanita Dalpiaz \\ Especialista en Desarrollo de Materiales Didácticos \\ Instituto de Cultura Brasil Colombia- IBRACO \\ ro_popa@hotmail.com \\ Luciana Stanzani \\ Candidata a Magíster en Educación con énfasis en Didáctica de Lenguas Extranjeras \\ Instituto de Cultura Brasil Colombia- IBRACO \\ stanzani.luciana@gmail.com
}

Resumen

Este artículo trata acerca del papel de un profesor extranjero en la enseñanza de un idioma extranjero a partir de la experiencia docente de dos brasileñas profesoras de portugués en un contexto de no inmersión en la ciudad de Bogotá. Constituye una conguración de los perles tanto del estudiante como del maestro inmerso en la enseñanza-aprendizaje del idioma portugués teniendo presente la relevancia sociocultural de la enseñanza de esa lengua, ya que ella hace parte de la construcción de la propia identidad de los brasileños como constituidores de una nación.

Palabras clave: portugués, lengua extranjera, enseñanza, profesor

\section{Summary}

This article is about the role of a foreign teacher in teaching Portuguese as a foreign language. It tells the experience of two Brazilian Portuguese teachers in the non - immersion context in the city of Bogota. It is a conguration of the proles of both the student and the teacher engaged in the teaching and learning processes of Portuguese, bearing in mind the cultural relevance of the language teaching, as it is part of the construction of identity of Brazilians as important part of their nation.

Key words: Portuguese, foreign language, teaching, tea-

\section{Introducción}

El objetivo de este trabajo es explorar un cuestionamiento acerca del papel del profesor de lengua extranjera, a partir de la experiencia como profesoras de portugués como Lengua Extranjera (PLE) a colombianos. Para cumplir con este objetivo, se desarrollará un perl de los estudiantes del Instituto de Cultura Brasil Colombia (IBRACO) y también se planteará una discusión, sobre la formación del profesor de PLE.

\section{El portugués para extranjeros: un breve panorama de su trayectoria}

Es importante decir que la enseñanza del idioma portugués como segunda lengua en

Ella tiene una larga historia de casi cinco siglos que se extiende desde los primeros esfuerzos de los religiosos jesuitas por la enseñanza del portugués a miembros de naciones indígenas (Almeida Filho, 1992). No obstante, no hay estudios e interpretaciones abundantes de esa tradición, porque no hay conciencia de su existencia, como tampoco de la importancia de estudios de cuestiones relacionadas con ellas. Desde este punto de vista es innegable, por lo tanto, la relevancia sociocultural de la enseñanza de

de la construcción de la propia identidad de los brasileños como constituidores de una nación. Así, estudiar y conocer esta tradición puede signicar conocer y comprender mejor la cultura y el carácter del pueblo brasileño.

Según apunta Gomes de Matos (1989), uno de los profesionales precursores en el trabajo de divulgación e institucionalización de esta 
área de enseñanza e investigación, el portugués como lengua extranjera empezó a desarrollarse a mediados de la década de los sesenta. De acuerdo con Almeida Filho (1992), a partir de la década de los sesenta y durante los años setenta se constató un mayor crecimiento de cursos de portugués y producción de materiales didácticos en instituciones en el exterior.

Ese aumento de interés en el idioma podría explicarse por algunas razones básicas. La primera de ellas se relaciona con el hecho que, con el desarrollo industrial de Brasil, se pasa a tener un mayor número de extranjeros que van a este país a trabajar, a través de empresas multinacionales interesadas en intercambiar, importar y exportar tecnología. Así, hubo un crecimiento continuo de la búsqueda de clases de portugués para extranjeros, principalmente en los grandes centros urbanos, se estimuló el interés de los profesores y también la proliferación de escuelas privadas que ofrecían cursos en esa especialidad.

La segunda razón se reere al aumento de los intercambios internacionales entre universidades brasileñas y del exterior, que aumentó también el número de personas interesadas en estudiar en el país, lo que llevó a las universidades nacionales a la creación de cursos regulares de PLE. Con ello creció, por lo tanto, la demanda de profesionales involucrados en el área de la enseñanza.

La tercera razón para el incremento del interés por el área de PLE sería la implantación del MERCOSUR por los gobiernos de Brasil, Argentina, Paraguay y Uruguay, que signica ampliación y mayor facilidad en las relaciones económicas, comerciales y de intercambio cultural con los países vecinos del sur de América Latina.

Se puede decir que la tradición de la enseñanza del portugués como lengua extranjera, aunque sea reciente, se aanzó desde de los años ochenta, al expandirse en áreas potencial- mente prósperas como la que se reere al mercado de trabajo y la del sector de la enseñanza.

\section{La enseñanza del portugués para extranjeros en la ciudad de Bogotá}

En primer lugar, hay que explicar el contexto de este tema de trabajo. Somos profesoras de portugués como lengua extranjera en el Instituto de Cultura Brasil Colombia (IBRACO) con sede en Bogotá. IBRACO es una entidad adscrita a la embajada de Brasil, que se dedica a la enseñanza del portugués desde 1996.

El IBRACO es el único organismo en Colombia autorizado por el Gobierno de Brasil para aplicar el examen de prociencia de la lengua portuguesa, Celpe-Bras ${ }^{1}$, desarrollado por el Ministerio de Educación de Brasil, aplicado allí y en otros países, como requisito para que el estudiante extranjero acceda a las becas que otorga anualmente el gobierno de ese país. Sin embargo, es oportuno mencionar también que en Medellín, EAFIT realiza dicha prueba.

En el Instituto trabajamos con un cuerpo docente básicamente conformado por profesores brasileños de diferentes áreas del conocimiento. El equipo está conformado por sociólogos, sioterapeutas, administradores, odontólogos, fonoaudiólogos y licenciados en lengua portuguesa, entre otros.

La población más signicativa del Instituto son estudiantes universitarios ya graduados que pretenden acceder a las becas otorgadas por el gobierno brasileño para las universidades de Brasil. Sin embargo, también hay estudiantes amantes de la cultura de Brasil, así como profesionales de distintas áreas en busca de mejores oportunidades laborales.

Teniendo en cuenta que el examen CelpeBras es de naturaleza comunicativa, es decir, evalúa la capacidad de uso de la lengua por medio de cuatro producciones escritas, en las

1 Para saber más sobre el examen, consulte http://celpebras.inep. gov.br/inscricao/ 
cuales el estudiante se enfrenta con diferentes géneros textuales (cartas, e-mail, folleto y artículos entre otros) e interlocutores, para vericar el dominio de la lengua en contextos formales e informales.

Para que el estudiante logre su objetivo de ser aprobado en el examen mencionado, IBRA$\mathrm{CO}$ ha trabajado con una metodología que se llama metodología híbrida (explicada más adelante), lo que se justica por lo novedosas que son todavía las teorías acerca de la enseñanza de portugués para extranjeros. Según José Carlos Almeida Filho (1997, p. 7), uno de los estudiosos que más se ha destacado en el área, "la enseñanza de portugués a hablantes de otras lenguas vive en una fase de consolidación institucional y de incremento de investigaciones en Brasil" (traducción nuestra). Aunque la lingüística aplicada a la enseñanza de portugués haya empezado en los sesenta (Almeida Filho, 1997), hoy se siente que se forman las bases para la enseñanza de tal idioma. Aparte de esto, el hecho de que estemos fuera del contexto de investigación de las universidades brasileras nos lleva a estudiar basadas en nuestra propia experiencia, o en la experiencia de otros académicos que trabajan allá.

De acuerdo a lo presentado arriba, el curso de portugués de IBRACO está destinado a jóvenes y adultos. Se compone de siete niveles, de 50 horas cada uno, y se ofrece como intensivo (entre semana) o los sábados. En el curso se trabaja con un libro didáctico, "Falar...Ler...Escrever... Portugues um curso para estrangeiros" (Sao Paulo, 2009) que integra el principal instrumento del método tradicional con la sistematización de las estructuras básicas de la lengua mediante la repetición de ejercicios escritos, básicamente, y con materiales de apoyo para cada nivel. Éstos fueron desarrollados por los propios docentes y están orientados al público especíco del Instituto, en una forma de trabajar con un abordaje comunicativo de la lengua por medio de actividades que incluyen lectura, video y música, así como producciones de textos orales y escritos.
Se entiende que, para la enseñanza de portugués a estudiantes que hablan español, no se puede aplicar una metodología únicamente comunicativa, porque eso podría hacer, a nuestro modo de ver, que el estudiante cayera en un estado de ínter lengua en el cual no alcanzara su total prociencia en la lengua portuguesa. De acuerdo con Almeida Filho (2001, p. 19),

"Hay ventajas indubitables en esa proximidad, especialmente si están combinadas con trazos afectivos como capacidad de riesgo, seguridad o también extroversión. Sin esos atributos, es de esperarse que ocurran las tentativas de obtención de uidez y de disponibilidad de vocabulario cuyo aparente (y posible) medio-éxito lleva a estancar la ínter lengua en niveles bajos de producción denominados popularmente de portuñol". (Traducción nuestra).

De acuerdo con esto, en la enseñanza de portugués a un hispano-hablante lo mejor es establecer las fronteras de las diferencias, para que el estudiante verdaderamente tome consciencia de que no se trata de la misma lengua. Sin duda, las semejanzas ayudan a que el proceso de aprendizaje sea más rápido, en comparación con estudiantes de otras lenguas.

\section{Resultados}

Dentro de ese universo aquí mencionado, podríamos cuestionarnos acerca del papel del profesor de PLE, especícamente en un contexto de no inmersión, y también sobre cuál sería su formación adecuada o esperada.

Los profesionales que llegan a IBRACO no son necesariamente de carreras relacionadas con las lenguas. Con este equipo interdisciplinario es viable pensar la lengua como un conjunto, en su contexto global, incluso en lo que se reere a la adquisición de vocabulario, sin limitar el aprendizaje a un grupo de reglas. Habida cuenta que los propios profesionales que estudiaron lenguas o lingüística no han tenido 
una preparación especíca para la enseñanza de su lengua materna a extranjeros, y, que este tema es muy reciente y todavía hay muchas universidades brasileñas que no lo han puesto en el currículo de sus cursos de lengua portuguesa, el Instituto elaboró un curso de formación de profesores, en el cual se habla de la metodología híbrida, y sobre algunos métodos que los profesores con más experiencia han aplicado con resultados satisfactorios.

Teniendo en cuenta al estudiante promedio del Instituto, esto es, aquel que habla español y por eso tendrá más facilidad en comunicarse, lo que como ya decimos puede caer en el riesgo de comunicarse con el "portuñol", pensamos que trabajar únicamente con un abordaje comunicativo no sería la mejor opción para él, porque no le daría la oportunidad de sistematizar las diferencias que hay entre el español y el portugués, que, en nuestra opinión, pueden ser resueltas con muchas propuestas que traen los libros más estructurales. También, tenemos que estar preparadas para este estudiante adulto, que estudia basado en la comprensión y que anhela saber el porqué deben actuar de una o otra manera, establecido en cada momento una relación con su lengua materna (Kunzendor , 1997).

Especícamente en la enseñanza de lenguas próximas, como es el caso del portugués y el español, creemos que trabajar con la gramática por la gramática puede ser una manera para que el estudiante, además de sistematizar las diferencias entre las dos lenguas, pueda hacer una reexión sobre su propia lengua.

Además concordamos con Lombello (1989, p. 45) en lo que se reere a la importancia del contenido gramatical en el aprendizaje de una lengua:

"Tanto por cuestiones de orden práctico como por la consideración de la arbitrariedad del signo lingüístico y de la necesidad del apoyo de sistematización al aprendiz, un programa general de enseñanza de una lengua extranjera para adultos debe ser orientado gramaticalmente, es decir, que debe tener una base gramatical organizada progresivamente en la cual se prevea una complementación incidental y variable a lo largo del desarrollo particular de cada programa". (Traducción nuestra).

El curso de formación de profesores también es el momento en el cual se reexiona sobre el papel del profesor de portugués como lengua extranjera a hispano hablantes. Es muy importante que el profesor conozca la lengua materna de sus estudiantes para poder entenderlos mejor en las dicultades presentadas. No podemos aceptar el posicionamiento de ciertas escuelas que deenden el principio de que, para enseñar portugués a extranjeros, basta ser un hablante nativo; hay que conocer también la cultura brasilera y actualizarse constantemente. En lo que se reere al uso de estructuras de la lengua hablada, un profesor que no esté en contacto constante con su país y con lo que se hace en la actualidad puede caer en el equívoco de enseñar expresiones coloquiales, por ejemplo, que ya no se usan o que son formas muy básicas, con las cuales el estudiante tendría dicultad de expresarse en el idioma en cuestión. Por eso, la formación pedagógica adecuada y las capacitaciones permanentes son fundamentales para la ecacia de cualquier curso.

Es evidente que no se postula aquí que el profesor deba ser del área de lenguas, pero es necesaria una preparación profesional, pues el éxito del aprendizaje de una lengua está relacionado sobretodo con la aplicación de nuevas teorías y con la capacidad de probar y de evaluar nuevos métodos.

\section{Conclusiones}

Por lo antes enunciado surge la necesidad de un curso de formación de profesores, para que los diferentes profesionales puedan actuar en el campo de la educación del PLE. El éxito 
del trabajo está en la aplicación adecuada del método y también en la constante actualización profesional, que posibilite al profesor un perfecto desempeño de sus funciones.

El profesor tiene un papel fundamental en la formación de un estudiante de lenguas extranjeras. Con tantos recursos tecnológicos con los cuales éste puede hoy en día aprender una lengua, sentimos que somos sus orientadores, pero sin contar con un contexto de inmersión, creemos en un profesor orientador

pues es con él que el extranjero mantiene su primer contacto y, consecuentemente, buscará orientaciones o informaciones sobre cómo, dónde y cuándo, es decir, se va a enterar, informar, conando, sobretodo, en sus recomendaciones [...] puesto que es él quien hará el puente entre el individuo extranjero y el nuevo mundo." (Souza, 2010, p. 105 - Traducción nuestra).

Porque al aportarles un poco de nuestra experiencia creemos que cuando se menciona el habla también se menciona la cultura. Dentro de ese contexto, el de un instituto cultural en el extranjero, una de las más ecaces cercanías a la cultura de Brasil es por medio de los profesores y de los programas, por ejemplo, que en el Instituto en cuestión se promueve para divulgar la cultura brasileña. En IBRACO se programa los "martes culturales", en los que se realizan presentaciones musicales, exposiciones, charlas con especialistas de diversas áreas como literatura, música y política.

En cada nivel, el estudiante puede aprender de la gramática básica a la avanzada, pero también desde la culinaria hasta la literatura de Brasil. Hay una programación de contenidos relacionados con la geografía y con la cultura de cada región de Brasil y el aporte del profesor es fundamental. IBRACO cuenta con profesores de muchos lugares de Brasil, lo que también favorece el contacto con la diversidad cultural existente en dicho país, que se sale de lo conocido internacionalmente a través de los medios o del turismo, por ejemplo. Aun con el hecho de que estemos en un contexto de no inmersión, es posible decir que los estudiantes del Instituto salen de sus cursos con un bagaje teórico con respecto a Brasil, su cultura y su historia.

Muchas veces por las películas, por la música y por todo este aparato que involucra la cultura y sus viajes a Brasil, el estudiante espera del profesor un lenguaje parecido al coloquial. Lo que se da en el Instituto, sin embargo, es un lenguaje más cercano a la lengua culta, ya que creemos que el tránsito de la lengua culta a la coloquial es mucho más fácil que de la coloquial a la lengua culta.

Además de orientar al estudiante en el aprendizaje de la lengua (entendemos que la cultura también hace parte de eso), el profesor es quien condiciona y motiva a sus estudiantes, ayudándolos a superar sus momentos difíciles.

Con respecto a ese contexto extranjero en el cual la lengua de los estudiantes es una lengua extranjera para los profesores, es posible decir que estar fuera del lugar de origen nos estimula a la reexión. Se analiza mucho más lo que pasa con el idioma y sobretodo con la cultura desde un contexto exterior. Esa abstracción nos hace trabajar todo el tiempo con el análisis de la comparación y nos estimula mucho a pensar en el otro y en la diferencia con el otro: la otra cultura, el otro idioma, el otro folclor y la otra música.

Finalmente, se enfatiza que nuestro objetivo, como profesoras del idioma portugués en Colombia, es hacer que el estudiante se acerque, a pesar de que no esté en un contexto de inmersión, a la cultura brasileña. Sea cual sea el método elegido para dictar un curso de portugués, es importante resaltar en que se aprende una lengua a través de la práctica constante de la misma. No existen fórmulas 
perfectas y absolutas, pues la lengua se caracteriza por su dinamismo. En este sentido, el mejor camino al aprendizaje es el de presentar propuestas innovadoras para la enseñanza en el momento actual, dejando abierta la posibilidad para nuevas modicaciones en futuro.

\section{REFERENCIAS}

Almeida Filho, José Carlos P. de; Lombello, Leonor C. (orgs.). (1997)O ensino de português para estrangeiros: pressupostos para o planejamento de cursos e elaboração de materiais. Campinas: Pontes.

Almeida Filho, José Carlos P. de. (1992). Relatório de Pesquisa: projeto interação e relevância no ensino de línguas Campinas: Unicamp.

Almeida Filho, José Carlos P. de. (2001). Português para estrangeiros interface com o espanhol. En: Almeida Filho, José Carlos P. de. (orgs.). Uma metodologia especíca para o ensino de línguas próximas? Campinas: Pontes.
Gomes de Matos, F. (1989). "Quando a prática precede a teoria”. En: Almeida Filho, José Carlos P. de.; Lombello, Leonor C. (orgs.). O ensino de português para estrangeiros: pressupostos para o planejamento de cursos e elaboração de materiais. Campinas: Pontes. Pp 11-17.

Lombello, Leonor C. (1989). "Quando a prática precede a teoria”. En: Almeida Filho, José Carlos P. de; Lombello, Leonor C. (orgs.). A anterioridade do lingüístico no planejamento de programas de ensino de segunda lengua. Campinas: Pontes. P 45.

Kunzendor , Júlia Cristina. Considerações quando ao ensino de português para estrangeiros adultos. En: Almeida Filho, José Carlos P. de.; Lombello, Leonor C. (orgs.). O ensino de português para estrangeiros: pressupostos para o planejamento de cursos e elaboração de materiais. Campinas: Pontes, 1997.

Souza, Ana Paula Kurpan de. O ensino de língua Portuguesa já foi além do limite: uma discussão teórica e metodológica sobre o ensino de PLE. Disponible en: http:// www.letras.puc-rio.br/publicacoes/ccci/Textos\%20 revisados/0\%20Ensino\% 20de\%20L\%C3\%ADngua\%20 Portuguesa\%20foi\%20al\%C3\%A9m\%20do\%20limite. pdf. Acceso: 4 mayo 2010.

Cómo citar este artículo:

Dalpiaz, R y Stanzani, L. Una experiencia contada: La enseñanza del Portugués como lengua extranjera a colombianos en Bogotá (2012). Espiral, Revista de Docencia e Investigación. 2, (1), 21 - 26 
